\title{
Féeries
}

Études sur le conte merveilleuX, XVII -XIX siècle

$12 \mid 2015$

À la croisée des genres

\section{Les Belles rendormies. Féeries fin-de-siècle}

Back-to-sleep Beauties: A Fin-de-siècle Extravaganza

\section{Sophie Lucet}

\section{(2) OpenEdition}

Journals

Édition électronique

URL : http://journals.openedition.org/feeries/974

ISSN : 1957-7753

Éditeur

UGA Éditions/Université Grenoble Alpes

Édition imprimée

Date de publication : 15 octobre 2015

Pagination : 131-153

ISBN : 978-2-84310-306-3

ISSN : 1766-2842

Référence électronique

Sophie Lucet, «Les Belles rendormies. Féeries fin-de-siècle », Féeries [En ligne], 12 | 2015, mis en ligne le 15 octobre 2016, consulté le 08 septembre 2020. URL : http://journals.openedition.org/feeries/974

() Féeries 


\section{LES BELLES RENDORMIES. FÉERIES FIN-DE-SIÈCLE}

La Belle au Bois qu'a réveillée

Le Beau prince franc Clodomir,

A préféré se rendormir

Dans la ronce et l'herbe mouillée.

Au sublime oubli résignée

Plutôt que vivre et souffrir,

La Belle a dit à l'Araignée :

"Tisse des toiles, Araignée.

L'ombre est douce à qui va mourir.»

Elle a préféré la charmante,

Reprendre son rêve enivrant Que vivre en notre âpre tourmente. Oh les grands iris noirs de ténèbres Au seuil des sept donjons funèbres De la Princesse au Bois Dormant. Jean Lorrain, Le Livre des légendes.

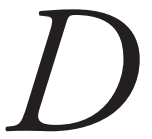

ANS SON ÉTUDE DE IOIO CONSACRÉE À LA FÉERIE2, Paul Ginisty - ancien directeur de l'Odéon et historien du théâtre - proposait en guise de préface à l'ouvrage un petit apologue sur le motif du don des fées, destiné à illustrer l'histoire d'un genre théâtral parvenu, selon lui, à un état de grande décadence. Les fées, jadis réunies, se seraient accordées — imaginait-il — à offrir aux hommes le secours du théâtre, pour alléger le fardeau de leur existence et leur permettre d'oublier momentanément leurs tristesses en cultivant sur le tréteau un art

I. J. Lorrain, «Petits contes de la forêt», dans Le Livre des légendes (sous la direction de J. des Gâchons), fascicule I, I895, p. 7-8.

2. P. Ginisty, La Féerie, Paris, Michaud, I9I0, p. 7-9. 
d'illusion et de mensonge bienfaisant, inspiré de la magie et des prestiges du royaume de féerie; mais la vieille fée acariâtre, malencontreusement oubliée parmi les convives et désireuse de se venger, aurait frappé ce beau projet de malédiction, en condamnant «un genre charmant fait pour tenter les poètes " à tomber dans les mains des vaudevillistes et autres "gens de métiers", et à perdre ainsi tout "esprit» dans les dévoiements d'un théâtre désormais préoccupé seulement de la matérialité du grand spectacle et des rentes du succès. Un tel diagnostic — on le sait — est le lieu commun d'une tradition critique texto-centrée qui, tout au long du XIX ${ }^{\mathrm{e}}$ siècle, tend à déplorer le manque de littérature des formes populaires du "théâtre oculaire", dont on voit pourtant mieux aujourd'hui combien elles ont été le vecteur de nouveaux régimes de théâtralité et de formes neuves ${ }^{3}$. Dans le même temps, à l'orée d'une étude portant sur la féerie, cette petite fable imaginée par son auteur présentait le double intérêt de rappeler, d'une part combien ce genre décrié — dont le succès ininterrompu a pourtant marqué tout le siècle — avait également su fasciner écrivains et artistes de tous bords, enchantés de ses conventions mêmes, qui semblaient si bien s'accorder avec les artifices inhérents à l'art du théâtre ${ }^{4}$, et d'autre part combien aussi les contes de Perrault, dès le XviII ${ }^{\mathrm{e}}$ siècle, et à l'origine des premières codifications du genre de la féerie (au moment de la Révolution), avaient fourni de manière privilégiée le matériau de toutes sortes d'adaptations scéniques et d'interprétations variées ${ }^{5}$; elles étaient rendues possibles par la plasticité d'un genre théâtral, par nature hybride, se définissant d'abord et avant tout par sa dimension spectaculaire, associant la parole et le chant, le théâtre et la danse, l'esthétique du tableau et la fantaisie visuelle, cultivant l'art du mouvement et de la métamorphose — avec tous les impératifs du «clou» — dans la variété des inventions plastiques et la succession de nombreux décors.

3. Sur le renouvellement de l'historiographie théâtrale sur ces sujets, voir notamment Orages, $\mathrm{n}^{\circ} 4$ (Boulevard du crime : le temps des spectacles oculaires), O. Bara (dir.), Gagny, APOCOPE, mars 2005; Le spectaculaire dans les arts de la scène, du Romantisme à la Belle-Époque, I. Moindrot (dir.), Paris, CNRS Éditions, 2006; R. Martin, La féerie romantique sur les scènes parisiennes (I79I-I864), Paris, Honoré Champion, 2007.

4. De Théophile Gauthier (Histoire de l'art dramatique en France depuis vingt-cinq ans, Paris, Hetzel, 1858-1859) à Mallarmé ("Crayonné au théâtre», Divagations, Paris, Fasquelle, I897), en passant par Jules et Edmond de Goncourt («Préface», Théatre, I879) et Émile Zola (Nos auteurs dramatiques, Paris, Charpentier, I88I), c'est une idée que l'on trouve formulée de manière récurrente chez plusieurs écrivains du XIx ${ }^{e}$ siècle, en dépit de leurs jugements négatifs sur l'état du théâtre contemporain et d'un art trop souvent voué aux facilités du divertissement.

5. Voir O. Piffault, Il était une fois... Les contes de fées, Paris, Seuil/Bibliothèque nationale de France, 200I, p. 559. 


\section{Belles de jours : scènes à succès et rêve littéraire}

La fortune remarquable du conte de "La Belle au bois dormant», auquel Ginisty se référait significativement dès cet apologue liminaire, paraît tout particulièrement représentatif du phénomène d'appropriation du conte de fées par le théâtre du XIX ${ }^{e}$ siècle, et d'acclimatation à la scène d'un type de récit propice à tous les déploiements du merveilleux scénique. De La Forêt enchantée ou la Belle au Bois dormant de Caigniez ${ }^{6}$ (sous-titrée "mélo-drame-féerie», qui figure parmi les premières adaptations du genre) à La Belle au bois-dormant, opéra-féerie de Clairville et Busnach de $1874^{7}$, les titres et les spectacles sont légion ${ }^{8}$, qui témoignent de ce travail d'adaptation, de transformation, de variations à l'infini dans la féerie, et des possibilités nombreuses de déplacement de sens, mais aussi de contamination générique que subissent les contes de fées sur les scènes de théâtre au XIX ${ }^{\mathrm{e}}$ siècle, dans la pure fantaisie, la légèreté, l'irrévérence parfois, au gré de la volonté des auteurs et librettistes, qui varient à l'envi sur la lettre des contes, sans craindre ni le burlesque ni l'anachronisme, dans un jeu d'amalgame et de recyclage généralisé : de la féerie au vaudeville, en passant par l'opéra, la féerie lyrique, le ballet, l'opéra-féerie, l'opérette, la comédie en vers ou en prose, les genres variés du théâtre à succès contribuent à ce jeu de réappropriation et de mise au goût du jour du «fonds Perrault», par une industrie du divertissement théâtral encline à tous les syncrétismes. De ces «féeries modernes» — qui feront ensuite les beaux jours du théâtre du Châtelet jusqu'en I9I4 - Ginisty ne pensait guère de bien, comme il l'écrit dans la suite de son ouvrage :

C'est l'une des premières interprétations scéniques du conte de Perrault, qui n'avait pas gagné, on s'en aperçoit sans peine à la lecture, à être arrangé par Caigniez. Que de Belles aux bois dormant se succéderont! C'est le thème qui sera le plus souvent traité, et qui, hélas, ne sera, comme il aurait dû l'être, réservé aux seuls poètes, faits pour

6. L.-C. Caigniez, La Belle au bois dormant, mélo-drame-féerie, mêlés de chants, danses et combats, musique du Citoyen Blanc, théâtre de la Gaîté, 25 Floréal, an VIII, Tiger, Paris.

7. La Belle au bois dormant, opéra-féerie en quatre actes, livret de Clairville et W. Busnach, musique d'H. Litolff, théâtre du Châtelet, 4 avril I874.

8. Quelques exemples dans la titrologie considérable des "Belles au bois dormant" scéniques: La Belle au bois dormant, féerie-vaudeville en deux actes, par MM. Bouilly et Dumersan (théâtre du Vaudeville, 20 février I8II); Saphirine ou le Réveil magique, mélo-féerie à grand spectacle, paroles de Merle et M. Ourry, musique de Leblanc (théâtre de la Gaîté, juillet I8II); L'Ogresse ou la Belle au bois dormant, vaudeville-folie-comi-parade, par M. Désaugiers et M. Gentil de Chavagnac (théâtre des Variétés, août I8II); Le Fils de la Belle au bois dormant, féerie en trois actes et douze tableaux, de Lambert-Thiboust, P. Siraudin et A. Choler (théâtre du Palais-Royal, août 1858). 
comprendre la beauté de son symbole. Que d'inventions saugrenues s'y mêleront, même avant longtemps qu'il passe, fatalement, dans les mains de Clairville?.

Le défaut de littérature du genre institué de la féerie est bien l'un des principaux reproches formulé dans le discours critique qui lui est consacré au XIX ${ }^{e}$ siècle, mais l'introuvable féerie littéraire est érigée en modèle souhaité d'un théâtre poétique digne de ce nom. L'œuvre dramatique de Théodore de Banville, pour partie demeurée dans le livre, de Diane au Bois ${ }^{10}$ au Forgeron ${ }^{\mathrm{II}}$ — avec l'exception notable de l'acte en vers du Baiser ${ }^{12}$ — constitue dans la deuxième moitié du siècle un point de cristallisation significatif de ces débats. Parmi ses comédies lyriques, son Riquet à la houppe, comédie féerique en quatre actes publiée sur le tard par le poète ${ }^{13}$, retient tout particulièrement l'attention : si l'œuvre s'inscrit dans le mouvement qui voit en France l'adaptation systématique des contes de Perrault au théâtre, elle manifeste l'ambition explicite de réconcilier poésie et théâtre, dans la forme d'un théâtre en vers étroitement associé au projet d'un merveilleux théâtral renouvelé par les pouvoirs du verbe créateur. La fable des amours du prince contrefait et de la belle princesse Rose, qui voit, par l'intercession des fées, l'esprit et la beauté se féconder mutuellement et échanger leurs qualités, constituait le moyen propice d'illustrer un tel projet, et l'action dramatique imaginée par le poète (constamment soutenue par un propos métalittéraire sans ambiguïté) est mise au service d'une célébration du lyrisme, à travers une fiction théâtrale clairement allégorique des pouvoirs de métamorphose de la poésie. La fée chez Banville — dans son théâtre comme dans ses poèmes et ses récits - est une figure récurrente, dont les pouvoirs thaumaturgiques constamment mis en scène participent à l'état d'euphorie poétique si caractéristique de l'œuvre du poète :

Ami, dit-elle, je suis la fée Ooh, une petite inspiratrice de chansons et de rimes, et c'est moi que la Reine a déléguée dans les comédies, pour induire les directeurs de théâtre à avaler quelquefois un peu de poésie, comme une médecine amère. Mais garde-toi bien de trahir mon incognito, car ces méchants-là me tueraient. Ne leur

9. P. Ginisty, ouvr. cité, p. 70.

Io. Th. de Banville, Diane au bois, comédie héroïque en deux actes, Paris, Michel Lévy frères, I864.

II. Th. de Banville, Le Forgeron, scènes héroïques, poème inédit, Paris, Maurice Dreyfous, I887.

I2. Th. de Banville, Le Baiser, comédie en vers, musique de P. Vidal, avec un frontispice de G. Rochegrosse, Paris, Charpentier, I888, représentée pour la première fois par A. Antoine (Théâtre Libre), au théâtre Montparnasse, le 23 décembre I887; et à La Comédie-Française, le 15 mai 1888.

13. Th. de Banville, Riquet à la houppe, comédie féerique en quatre actes, Poèmes inédits, Paris, Charpentier, I884. 
fais pas soupçonner qu'ils ont affaire à une pensée vivante, et laisse leur croire éternellement que je suis une poupée de bois ${ }^{14}$.

Auxiliaire indéfectible d'un idéalisme esthétique constamment reformulé, la fée chez Banville est le signe de l'absolu poétique, dont son œuvre figure la quête, et dont ses admirateurs - parnassiens, néoromantiques et symbolistes confondus - célèbrent le rayonnement dans ses textes. Au théâtre, le merveilleux banvillien se traduit dans la double expression d'une nostalgie d'un âge d'or perdu, et dans la croyance en la réactivation des pouvoirs de la poésie à l'époque moderne. Aussi n'est-il guère surprenant qu'il ait pu penser, dans un projet d'opéra lyrique cette fois, à concevoir un livret pour une Belle au bois dormant, comme nous l'apprend une lettre de I890 adressée au compositeur Jules Massenet : «Il me semble que le célèbre conte de : La Belle au Bois dormant nous donnerait tout ce que nous cherchons. Voulez-vous que j'essaie un plan sur ce sujet ?' peut rêver à ce qu'aurait donné une telle collaboration, pour laquelle il n'existe apparemment pas de documents préparatoires. Le motif du don des fées, celui du sommeil séculaire, la quête du prince, et son baiser, le réveil merveilleux de la belle endormie, tous ces attendus du conte auraient certainement été exploités avec brio par le maître des fantaisies inspirées ${ }^{16}$, dans le souci de donner forme et puissance à la "beauté de son symbole», pour reprendre l'expression de Ginisty — et au travers d'une interprétation du conte de Perrault qui aurait sans nul doute privilégié la dynamique d'une reviviscence de l'ancienne poésie, et celle d'un ré-enchantement du monde par les pouvoirs de l'art, littérature et musique confondues. Mais sans doute aussi Banville, disparu l'année suivante, avait-il d'ores et déjà renoncé à un projet rendu banal, ou périlleux, par le très grand nombre des «Belles au bois dormant» dramatiques et lyriques du XIX ${ }^{\mathrm{e}}$ siècle ${ }^{17}$, et

I4. Th. de Banville, "Les Huit sous de Pierrot», Contes féeriques, Paris, Charpentier, I882, p. I8I.

I5. Lettre du 5 septembre 1890, citée par J. de Palacio, dans Les Perversions du merveilleux, [Paris], Séguier, I993, p. I42. Ponctuation conforme à l'original.

I6. Dans l'esprit, par exemple, de cette strophe de "La Ville enchantée» des Odes funambulesques (Paris, Poulet-Malassis et De Broise, I857, p. 218) :

«La Belle au bois dormant, sur la moire fleurie

De la molle ottomane où rêve le chat Murr,

Parmi l'air rose et bleu des feux de la féerie

S'éveille après cent ans sous un baiser d'amour."

17. La Belle au bois dormant, opéra en trois actes, paroles d'E. Planard, musique de Caraffa, théâtre de l'Opéra, I825; ballet de Scribe et Herold en I829; féerie de G. Gavrard en I831; drame en cinq actes et sept tableaux d'O. Feuillet en I863; opéra-féerie de Clairville, Busnach et Littolff en I874; La Belle au bois dormant, opérette en trois actes, paroles d'A. Vanloo et G. Duval, musique de Ch. Lecoq sur des thèmes de J. Offenbach, Bouffes Parisiens, I9 février 1900. 
par les représentations convenues à cette époque, d'un idéalisme poétique, dont le réveil de la Belle au bois dormant constituait l'un des poncifs. En 1907, c'est à l'un de ses amis et admirateurs ${ }^{18}$, en la personne du poète Jean Richepin, qu'il reviendra, de fait, de contribuer au rêve d'une "féerie totale ", dans laquelle la littérature devait entrer à égalité avec les ressources de la machinerie et celles de la musique, pour revivifier le genre même de la féerie théâtrale. Pièce à grand spectacle, réalisée avec des moyens considérables au théâtre Sarah Bernhardt, avec la collaboration du dramaturge et librettiste Henri Cain (lequel avait collaboré dix ans plus tôt, rappelons-le, à la Cendrillon ${ }^{19}$ de Massenet), celle du compositeur Francis Thomé, des décorateurs connus (Bertin, Jambon, Amable...), une foule d'acteurs, chanteurs, danseurs, choristes, figurants de toutes sortes, cette féerie lyrique, d'une ambition littéraire affichée et thématisée, prétendait répondre doublement aux attentes d'un public épris de merveilleux scénique et à celles des tenants d'un théâtre littéraire hérité du rêve banvillien. Cette Belle au bois dormant ${ }^{20}$, féerie lyrique en alexandrins alternant les parties parlées et chantées, obtint un très vif succès auprès du public et dans la presse, avec de nombreuses représentations. Visant une mise en perspective de la puissance des contes, à travers un travail d'adaptation très libre et d'audacieux effets de mises en abyme, elle condensait certains traits des «Belles au bois dormant» théâtrales du XIX ${ }^{\mathrm{e}}$ siècle, désignant ce conte comme le sujet par excellence d'un théâtre poétique : construite autour de Sarah Bernhardt — bonne fée d'un spectacle dont elle était à la fois le metteur en scène et l'actrice principale -, l'action en douze tableaux jouait d'emblée sur le double motif du «réveil des contes bleus», dans un monde qui ne croyait plus au merveilleux, et sur celui de la Belle endormie, autour de la figure dédoublée du poète et du Prince Landry, incarnée par la comédienne dans un rôle travesti comme elle les affectionnait. Du conte primitif étaient retenus les principaux moments (le baptême, le fuseau, le château du sommeil, l'endormissement général, le retour du prince, ses épreuves pour rejoindre le domaine enchanté, le baiser, le réveil...) tandis qu'était omise la vengeance de la belle-mère ogresse, conformément aux usages dominants des adaptations théâtrales

18. Voir J. Richepin, «Banville au Luxembourg : Ode à Banville», La Quinzaine bourbonnaise, 1892, p. 224-225.

19. Cendrillon, conte de fées en quatre actes et six tableaux, d'après Ch. Perrault, musique de J. Massenet, création à l'Opéra-Comique le 24 mai 1895.

20. J. Richepin et H. Caïn, La Belle au bois dormant, féerie lyrique en vers, en un prologue, deux parties, quatorze tableaux, musique de scène par M. Fr. Thomé, Paris, Imprimerie de «l'Illustration", 1907. 
du conte au XIX siècle $^{2 \mathrm{r}}$; à partir de là, les poètes et le musicien avaient déployé un poème qui portait en écrin le texte de Perrault, tout en élargissant explicitement ses perspectives en direction d'un drame allégorique des pouvoirs de la poésie et de l'éternité de la vie de l'esprit et de l'idéal. Nouvelle Miranda à la découverte du monde, la belle rencontrait le poète Landry avant l'épisode du fuseau, et lorsqu'elle sombrait dans le sommeil séculaire, c'était à lui que la fée marraine annonçait l'avenir et les termes d'une mise à l'épreuve, contre le temps et contre l'oubli, du serment d'amour échangé. Le centre de gravité de la pièce déplacé sur la figure du poète, la deuxième partie de la féerie mettait en scène le miracle de sa réincarnation en prince de légende, littéralement opérée par le souvenir d'un conte de fée. Le périple difficile qui suivait, dans des tableaux relevant du grand spectacle et de la quête allégorique, conduisait le prince à affronter le dragon dans "le bois des épouvantes", à résister aux sortilèges d'une démone, aux appels mortifères de languides ondines, avant de franchir en marchant sur les eaux le "lac de désespérance», moment miraculeux préludant au baiser puis au réveil, et à la reconnaissance extatique du poète par la Belle. Typique des syncrétismes du théâtre fin-de-siècle et de la Belle Époque, le poème dramatique conçu par Richepin et Cain et la mise en scène fastueuse de Sarah Bernhardt faisait ainsi feu de tout bois, mobilisant les références et les esthétiques les plus variées, depuis la féerie animalière du prologue, la comédie joyeuse et les anachronismes poétiques des premiers tableaux, les références au merveilleux shakespearien et banvillien, les effets de lyrismes romantiques, le drame et l'allégorie, jusqu'aux réminiscences wagnériennes (Siegfried, Parsifal) de la quête du prince, etc. Constituant un incontestable moment de récapitulation thématique et esthétique dans l'histoire des Belles au bois dormant au théâtre, la féerie de 1907 , non sans maniérisme, variait sur le motif d'un idéalisme poétique largement répandu au théâtre et dans la littérature depuis l'époque romantique. Le charme de l'ancienne féerie s'y trouvait-il réactivé pour autant, et la poésie miraculeusement "réveillée», revivifiée au théâtre, conformément au programme thématique de la pièce? En dépit du concert des louanges

2I. L'aspect cruel et monstrueux de la deuxième partie du conte de Perrault est traité dans certaines adaptations, au début de l'âge de la féerie notamment (voir par exemple L'Ogresse ou la Belle au bois dormant, vaudeville-folie-comi-parade en un acte, de Désaugiers et Gentil de Chavagnac, théâtre de Variétés, 28 août I8II), plus volontiers dans certaines versions burlesques du récit - et ce à l'encontre de la tendance majoritaire des adaptations théâtrales de «La Belle au bois dormant ", lesquelles œuvrent en général à l'euphémisation ou à la neutralisation, par omission, de la monstruosité et de la violence du conte, en faveur d'un merveilleux orienté vers le dénouement poétique et miraculeux du réveil de la belle endormie, désormais conçu comme un point d'acmé de la fable. 
d'une partie de la critique toujours prête à encenser Sarah Bernhardt, il est permis d'en douter, si l'on retient plutôt l'effet de saturation de références de cette féerie trop savante, dont l'esthétique tape-à-l'œil et foncièrement kitsch s'adaptait habilement à l'air du temps, recyclant superficiellement et de manière décorative certains thèmes et motifs caractéristiques de l'imaginaire fin-de-siècle, entre pessimisme schopenhauerien et langueurs symbolistes (ce que ne manquèrent pas de relever des observateurs plus attentifs).

Le motif d'une Belle inapte au monde moderne et qui choisit de se rendormir en tournant le dos à une réalité décevante est devenu, de fait, le cliché d'une fin-de-siècle qu'effraient les mutations de la vie moderne : on découvre ainsi l'expression vulgarisée de cette mélancolie belle-époque en 1902 dans un spectacle d'ombres de Lucien Métivet, féerie chantée en dixneuf tableaux lumineux, qui fut représenté au théâtre des Mathurins. Cette Belle au Bois-Dormant ${ }^{22}$, traitée à la manière d'un lumino-conte - dans le genre joyeusement syncrétique qui fit en I888 le succès de La Tentation de Saint-Antoine de Georges Fragerolle et d'Henri Rivière ${ }^{23}$ au Cabaret du Chat-Noir - montrait un prince moderne qui venait à bicyclette réveiller la princesse des légendes. L'invitant à quitter «le pays mensonger du rêve», il l'emmenait parcourir le monde moderne lui découvrant le spectacle de "la forêt coupée», des usines, de la "ville moderne», des trains, des cuirassiers, etc. Cette réalité faite de laideur et de violence, la Belle s'empressait de la nier, en abandonnant là son «moderne» prince charmant, et en retournant dormir dans son palais merveilleux :

La Belle a frissonné devant le ciel Tragique

Et son cœur s'est gonflé d'un douloureux émoi

Ô, Prince, je retourne en ma forêt magique.

Votre siècle moderne est trop nouveau pour moi ${ }^{24}$.

\section{Belles de nuits : poèmes et féeries symbolistes}

Les belles endormies ont notablement inspiré les poètes des années I890, constituant l'un des emblèmes privilégiés de la quête poétique des symbo-

22. L. Métivet, La Belle au Bois-Dormant, féerie chantée en Ig tableaux lumineux, poème et images de L. Métivet, musique de J. Vieu, Paris, Enoch et $C^{\text {ie }}$, Flammarion, I9O2.

23. G. Fragerolle, La Tentation de Saint-Antoine. Féerie à grand spectacle en 2 actes et 40 tableaux, musique nouvelle et arrangée d'A. Tinchant et G. Fragerolle, dessins de H. Rivière, Paris, Plon, Nourrit \& C Cie, s.d. [1888]. Voir S. Lucet, Le Théatre en liberté des symbolistes, dérives de l'écriture dramatique à la fin du XIXe siècle, thèse nouveau régime, sous la direction de J. de Palacio, Paris IV Sorbonne, 1997, p. 236-244.

24. L. Métivet, ouvr. cité, p. 42. 
listes, et un motif récurrent, dont les valeurs ambivalentes modulent autour de l'extase du rêve des nostalgies indicibles, des regrets de l'inaccompli, de la mélancolie de la perte. La légende de la "Belle au Bois-dormant» semble offrir, en outre, le cadre idéal d'un dialogue du poète avec sa muse, constituant le vecteur d'une théâtralité singulière à l'œuvre dans certains textes que les poètes symbolistes du groupe de La Revue indépendante et du Mercure de France ont composés sur ce thème, et qui semblent curieusement se faire écho les uns aux autres. Image de la beauté idéale, symbole d'un lointain inaccessible et d'un passé à jamais clos sur lui-même, la «Belle» est ainsi «motif de mélancolie» dans les Poèmes anciens et romanesques d'Henri de Régnier, à travers le triptyque de sonnets intitulé «La Licorne», évoquant, dans la séquence des trois titres, le drame de l'impossible réveil : «Et la Belle s'endormit», «Et le Chevalier ne vint pas», «Et la Belle mourut $»^{25}$ : abandonnant toutes les circonstances du conte pour ne retenir que le motif du sommeil de cent ans, le premier sonnet esquisse le tableau de la belle endormie, veillée par une licorne, sentinelle du "vaste songe " où se trouve la princesse, et présente ainsi une double image de pureté et d'inaccessibilité, évocatrice aussi bien des figures mystérieuses de Gustave Moreau que du rêve glacé de l'«Hérodiade» de Mallarmé. Dans le second sonnet, s'approfondit l'intensité du vide, de la solitude et du silence mortifère autour de la Belle qui dépérit, et en l'absence du Héros qui eût pu "vaincre d'un baiser le magique sommeil noir ${ }^{26}$ ». Cette irrémédiable absence conduit, dans le troisième sonnet, au motif de la fuite effrénée et terrifiée de la licorne, «folle d'avoir flairé les mains froides de $\operatorname{mort}^{27}$ ", et en allée loin des paysages glacés, où la Princesse est morte. Dans les Chansons d'amant de Gustave Kahn, le sommeil de la Belle n'est plus tant un état de léthargie proche de la mort qu'un état de conscience et d'existence paradoxal, manifestant le choix d'une sécession du réel; elle est alors "la Belle au Château rêvant ${ }^{28}$ ", l'héroïne de toutes les odyssées du songe, celle qui ne répond pas aux prières du Pèlerin et supplie que le sommeil et le rêve la reprennent :

LA BELle :

Reviens à moi, sommeil, scelle-toi sur ma bouche

Des mirages de leurs visages garde le lac de mes yeux

25. Henri de Régnier, Poèmes anciens et romanesques, Paris, Librairie de l'Art Indépendant, I890, p. 70-72. (La suite de ces trois sonnets fut d'abord publiée dans La Wallonie, février 1889, p. 67-69.)

26. Ibid., p. 7I.

27. Ibid., p. 72.

28. G. Kahn, «La Belle au château rêvant», Chansons d'amant, Bruxelles, Lacomblez, p. 9-25 (pré-originale en $\mathrm{I} 887$, dans La Revue indépendante). 
Reprend-moi dans le val aux mousses quiétantes Où toujours l'amoureux soulève un pan de tente Et se retire peureux $[\ldots]$

Les meurtres de ma vie enclos-les dans tes deuils Le méfait de ma beauté couvre-le d'un pan de la nuit Donne le fleuve d'oubli qui berce et s'abolit

Ah! reprends-moi, sommeil, scelle-toi sur ma bouche

Et tandis que sort le Pèlerin, que le château retombe dans le mutisme séculaire, le veilleur des tours chante ${ }^{29}$.

Le long poème dialogué de Kahn, déployé en vers libres, évoque ainsi une sorte de "palais mental» où résonnent des voix contradictoires («le Veilleur», "le Pèlerin», "Le Mage», "La Belle»), comme l'écho d'un drame intérieur, celui d'une conscience malheureuse aspirant à l'absolu, mais incapable de susciter "la lancinante dormeuse hantante de [son] rêve $^{30}$ ". André-Ferdinand Hérold, à son tour dans ses Chevaleries sentimentales $^{31}$, consacre à la «Belle au Bois Dormant» un poème en forme de dialogue, qu'il dédie significativement à Pierre Quillard, comme en réponse à un texte sur le même thème, publié par ce dernier en I890, et intitulé "Prologue pour un poème dialogué ${ }^{32}$ " (lequel ne fut manifestement jamais composé). Dans ce poème narratif et choral en trois parties — qui célèbre tout d'abord le mystère et la quiétude du sommeil de la Belle «loin des cris sourds de haine et de colère, Loin des hommes tremblants et voués aux douleurs" - , le motif du réveil se décline moins sur le mode de la mélancolie que sur celui d'une révélation confirmant les pouvoirs du rêve : si le prince parvient à éveiller la Belle, c'est pour mieux rejoindre dans le royaume des songes et de l'idéal celle que son âme a pressentie; lui, le «royal Solitaire», «le Pur», au bout de sa quête devient le "Renonciateur", qui dédaigne la terre, les «luttes vaines", "les âpres désirs", et peut dès lors conquérir la Belle, dans l'extase finale d'une fusion amoureuse et lyrique. Au rebours de cette mystique du rêve, et pour citer un autre exemple des variations symbolistes nombreuses autour du conte de «La Belle au bois dormant», le poème (un simple fragment) du sym-

29. Ibid., p. 24.

30. Ibid., p. I6.

31. A.-F. Hérold, «La Belle au Bois Dormant », Chevaleries sentimentales, frontispice d'O. Redon, Paris, Librairie de l'Art Indépendant, I893, p. I63-174 (pré-originale dans Le Mercure de France, février I892, p. I25-I29).

32. P. Quillard, «Prologue pour un poème dialogué», La Wallonie, I890, p. 339-340 : cinq strophes d'alexandrins évoquent la vision merveilleuse d'une belle endormie au bord d'un lac, éveillée par un chevalier venu du pays des rêves, pour ranimer la «blonde princesse aux yeux pers». 
boliste belge Fernand Séverin, publié en I894 dans Le Coq rouge, semble répondre ironiquement à celui d'Hérold, en formulant la réponse d'une Belle qui éconduit purement et simplement l'intrus venu la distraire de ses songes :

Exulte, si tu veux, de ta conquête vaine.

Mes yeux ont parcouru les monts, les bois, les plaines :

Tout ce monde imprévu dont tu m'offres la fleur

N'aura rien de si doux qu'ils n'aient rêvé meilleur. [...]

Mon âme, ô conquérant, te restera voilée :

Le songe bienheureux dont m’as réveillée

Mettra, quoique je fusse, entre elle et ton baiser

Un voile d'inconnu qu'il ne saurait briser.

Car ceux, qui comme moi, captifs d'un charme antique,

Ont sommeillé cent ans dans le palais magique,

Ne sauraient délaisser ces limbes merveilleux

Lorsqu'un reflet divin s'en prolonge en leurs yeux. [...] $]^{33}$

Le thème du sommeil séculaire et celui d'un réveil problématique ouvrant sur un tragique exil sont ainsi les traits essentiels que retiennent les symbolistes, dans ces relectures variées, synthétiques et abstraites de la légende, qui célèbrent les valeurs du rêve et de l'idéal face à une réalité douloureuse et décevante, et sondent avec nostalgie l'énigme du songe de la Belle des légendes. Ces poèmes participent à l'élaboration d'un imaginaire symboliste spécifique autour de la Belle au bois dormant, contribuant à fixer un lieu commun, dont on peut découvrir les avatars mondains dans deux adaptations du conte de Perrault, destinées l'une et l'autre à des représentations d'avant-garde dans le contexte de l'expérimentation symboliste au théâtre : Le Songe de la Belle au Bois, "conte de fées en 5 actes, en prose», de Gabriel Trarieux ${ }^{34}$, dont Maurice Denis brossa les décors en I892 pour une représentation privée dans le salon des Finaly ${ }^{35}$, et La Belle au Bois dormant, "féerie dramatique" de Henry Bataille et Robert d'Humières $^{36}$, représentée le 24 mai 1894 au Casino de Paris, par le Théâtre de l'Euvre de Lugné-Poe, et sans doute l'un de ses échecs les plus cuisants.

33. F. Séverin, «La Belle au Bois» (Fragment), Le Coq rouge, n 8-9, décembre I894-janvier 1895 , p. 390.

34. G. Trarieux, Le Songe de la Belle au Bois, conte de fées en cinq actes, Paris, Librairie de l'Art Indépendant, 1892.

35. Voir J.-P. Bouillon, Maurice Denis, Genève, Skira, 1993; Maurice Denis (1870-1943), catalogue de l'exposition de Lyon-Cologne-Liverpool-Amsterdam, 1994-I995, Paris, RMN, 1994.

36. H. Bataille et R. d'Humières, La Belle au Bois dormant, féerie dramatique en trois actes, représentée par Lugné-Poe au Nouveau Théâtre (théâtre de l'Cuvre), le 24 mai 1894, musique de G. Hue, décors de Rochegrosse et Auburtin, exécutés par M.-L. de la Quintinie, costumes d'après 
Non sans parenté et trahissant l'effort de très jeunes poètes sous influence, ces deux contes théâtraux modulent, en effet, chacun à sa façon les motifs principaux d'une interprétation symboliste du conte de Perrault, faisant sourdre l'inquiétude, l'angoisse et la mélancolie au cœur du merveilleux, et tirant irrésistiblement la féerie du côté du tragique. Ainsi, les deux pièces orientent-elles d'emblée la fable vers les circonstances d'un réveil douloureux de la Belle, reléguant dans un avant très vaguement légendaire les prémisses du conte. L'enjeu ne se situe plus, dès lors, dans les termes d'une malédiction conjurée, mais dans la découverte difficile d'un réel inhospitalier, hostile, et dans l'incurable nostalgie qu'éprouve l'héroïne du sommeil et du songe dont on l'a exilée.

Le Songe de la Belle au Bois de Trarieux - significativement publiée en I892 à la Librairie de l'Art indépendant, lieu d'édition de prédilection de la poésie symboliste et décadente — s'ouvre ainsi sur le tableau de la Belle endormie, dont l'énigme, à l'orée de la pièce, avait incontestablement de quoi tenter le nabi Maurice Denis. En dépit de la déploration d'une nature environnante jalousement complice et gardienne, les temps semblent venus de la fin de l'enchantement, rompu par un prince Azur qui se dérobe tout aussitôt, annonçant le moment de l'épreuve nécessaire et de la rencontre avec «la vie anxieuse ${ }^{37} »$. Seule, à son réveil, la Belle doit affronter alors, dans la solitude et l'angoisse, son retour dans le palais triste d'un très vieux roi, en qui elle peine à reconnaître son père de jadis, à qui il revient de proférer les paroles d'impuissance d'un vieillard maeterlinckien :

Si! si! Quelque chose lui manque... Je ne peux plus le deviner, je suis trop vieux... elle n'a pas souri depuis son retour; elle ne veut plus voir personne... Elle n'est pas comme les autres filles que leurs mères ont élevées... Je crois qu'elle a dormi trop longtemps sous les arbres... Ne dirait-on pas un songe de clairières au fond de ses yeux?... Et puis, elle ne parle jamais que toute seule, ou avec cette Elsa qui couchait à ses pieds, dans son sommeil... Je crois que les fées à son berceau lui ont fait des présents étranges... c'est une singulière petite fille, elle a toujours l'air étonnée... mais pour le comprendre je suis trop vieux ${ }^{38} \ldots$

Irrémédiablement étrangère, à la manière des Maleine et Mélisande, la petite princesse de Trarieux n'a de cesse dès lors de retrouver son rêve perdu, se dérobant à tous les hommages, à la royauté dont elle hérite, aux fastes du monde. À la fin de la pièce, la princesse en fuite, à bout de force,

Burne-Jones et Rochegrosse; pièce non publiée. Copie manuscrite aux Archives nationales [voir Censure dramatique, pièces jouées dans les théâtres et les cafés concerts, F. I8 I242].

37. G. Trarieux, ouvr. cité, acte I, scène 2, p. I4.

38. Ibid., acte II, scène I, p. 24. 
retrouve le "bois magique», aspirant de tout son être au rendormissement et à l'oubli, dans la pensée d'un renoncement auquel l'arrache in extremis le Prince Azur, miraculeusement ressurgi, en place d'un mendiant à qui elle a donné à boire, et la conduisant cette fois vers la vie retrouvée et vers un nouvel espoir. Alternant dialogues en prose et poèmes choraux (où parlent les arbres, les femmes sur la mer, les oiseaux...), l'essai de théâtre poétique et symbolique de Trarieux - qui démarque constamment le théâtre de Maeterlinck, et fait signe aussi vers Wagner et Villiers de l'IsleAdam (le fidèle serviteur à qui le roi confie sa fille se nomme Axel) — relie ainsi significativement la fable de la Belle au bois dormant à la thématique spiritualiste du réveil de l'âme : deux fois libérateur, le Prince Azur devient une figure d'initiateur, dans un processus qui conduit à une authentique libération spirituelle. Non sans ambiguïté cependant, malgré ce final heureux, Le Songe de la Belle au Bois de Trarieux — qui fait mourir l'oiseau bleu des contes à la fin du troisième acte ${ }^{39}$ - se déploie dans la tonalité mineure d'une féerie paradoxalement désenchantée et empreinte de toutes les nostalgies fin-de-siècle.

Essai revendiqué d'un théâtre poétique et métaphysique, La Belle au bois dormant, féerie littéraire et décorative du jeune Henry Bataille — qui ne publia jamais ce texte de jeunesse ${ }^{40}$ — affiche les maniérismes d'un spectacle aspirant à la «féerie d'art». Le poète, qui empruntait au symbolisme ses thèmes et ses motifs, tout en rêvant d'éblouir une scène à succès, avait de fait commencé par proposer sa pièce à Sarah Bernhardt, ce à quoi il avait dû renoncer car l'actrice lui avait demandé trop de coupures et de changements ${ }^{4 \mathrm{I}}$. Peintre et poète lancé dans le monde, Bataille demanda à Lugné-Poe une sorte d'association pour un spectacle où il entendait prendre en charge une partie de la mise en scène, ainsi que le souci des décors (confiés à Georges Rochegrosse ${ }^{42}$ ) et des costumes (inspirés par le peintre préraphaélite Burne-Jones). Il s'était en outre assuré la colla-

39. À la fin du troisième acte, le petit Yvon — dans le cousinage des enfants maeterlinckiens, le petit Allan, dans La Princesse Maleine, le petit Yniold, dans Pelléas et Mélisande — vient annoncer en pleurant la mort de son "oiseau bleu»: "Je ne veux plus avoir d'oiseau!» (G. Trarieux, ouvr. cité, p. 5I-52)

40. Pour un résumé analytique plus détaillé de cette pièce inédite, dont une copie manuscrite se trouve aux Archives nationales, voir S. Lucet, Le Théatre en liberté des symbolistes, ouvr. cité, p. I68-I80.

4I. Voir la lettre adressée par Bataille à Lugné-Poe, et citée par ce dernier dans La Parade, souvenirs et impressions de théâtre, t. 2 Acrobaties (I894-1902), chap. 5 "Un client sérieux : Henry Bataille", p. $82-83$.

42. G.-A. Rochegrosse (1859-1938), peintre et illustrateur, était le beau-fils de Th. de Banville (qui lui dédia Les Exilés). 
boration de Robert d'Humières, aristocrate et officier de cavalerie (caution mondaine de l'entreprise), et put ainsi réunir des moyens bien plus importants que ceux dont disposait habituellement Lugné-Poe, pour la réalisation de sa féerie. La représentation constitua de fait un événement mondain, qui déplut aux symbolistes qui y virent une dérive fâcheuse du théâtre de l'CEuvre, et une caricature de l'idée théâtrale symboliste.

La trajectoire de cette pièce ambitieuse se déploie, comme chez Trarieux, entre le moment du réveil et celui du rendormissement de la Belle, mais dans une toute autre tonalité, emphatiquement explicative ici, et compliquée par une véritable mise en intrigue du scénario inversé du conte. Heurt de la réalité décevante, nostalgies du songe, présent désenchanté, idéal menacé, incompatibilité du merveilleux et de la modernité, inéluctable désertion des fées... - tous ces motifs de l'idéalisme et du pessimisme fin-de-siècle s'y trouvent savamment orchestrés et remotivés par les artifices de la fable théâtrale. Non seulement l'éblouissement, mais aussi les anxiétés du réveil de la Belle occupent longuement un premier acte qui s'emploie à activer tout le potentiel théâtral de ce point d'acmé du conte de Perrault : apparitions féeriques, contemplation et baiser, transformation à vue du décor, duo lyrique... La nourrice de cette princesse "de mille ans" y chante en filant son rouet, image de Parque ou de "Gardienne ", à l'orée d'une féerie à haute vocation symbolique. À la manière de la vieille reine des Sept Princesses de Maeterlinck, elle s'inquiète aussi de voir le Prince approcher le sommeil de la Belle enchantée :

La VIeIlle (lui montre le passage) :

Arrête... attends... j’ai peur... Songe que cette minute est le dénouement de mon immémorable attente. Prince, si la porte allait s'ouvrir. Oh! ce n'est pas possible que cela soit vrai. Attends... Si elle était morte ta fiancée... Doucement, plus doucement... Ne fais pas crier la serrure... Tu vas la réveiller... Et puis il faut que je te dise encore... je n'ai pas fini... ce n'est qu'au troisième baiser de ta bouche qu'elle se lèvera... Les fées l'ont dit ${ }^{43}$.

Le moment de béatitude passée, dans la rencontre archétypale de l'homme et de la femme - traitée à la manière de l'Antonia de Dujardin — l'inquiétude s'empare de la Belle, qui fait entendre à son tour l'expression du malaise et de l'irrémédiable sentiment d'étrangeté des princesses de Maeterlinck :

Je suis une pauvre petite princesse, une enfant, quoique j'aie dormi près de mille ans et rêvé bien des rêves. [...] Oh! comme je suis seule! nulle chose à présent n'est plus

43. H. Bataille et R. d'Humières, ouvr. cité, acte I, scène 3. 
de mon âge! Mes compagnes d'autrefois sont mortes, voilà bien des années... Je suis comme abandonnée dans une île. [... ${ }^{44}$

L'acte s'achève sur une tirade mélancolique de la fée, venue unir les jeunes gens avant leur retour dans la réalité, formulant un adieu dont les termes explicitent les enjeux de la pièce, soit la survivance problématique de la poésie, dans un monde où elle n'était plus qu'une présence diffuse, perceptible seulement à des élus :

Je vous unis, au nom de votre antique espoir et de votre jeune amour. Retournez à la vie. Que votre passion vous promène, nef aux voiles de soie, sur le flot inquiet du siècle. Je veux que vous communiiez en toutes les modernités. Vous serez heureux, vous devez l'être : un rare trésor vous est échu à l'un et l'autre. Et toi, mon enfant, sois bénie. Tu m'es chère pour toi-même, et aussi parce que c'est toi le dernier gage de pouvoir que m’a laissé le Destin. Ta vie est la mienne. Toi partie, je me dissoudrai dans le vent, l'écume de la vague, la fumée des villes sombres. Je ne puis mourir. Je serai l'immense poésie éparse. C'est de m'avoir devinée que, les soirs, aux fenêtres ou sur les collines, les jeunes-hommes resteront devant la Nature, la tête dans les mains et le coeur comme un tabernacle. Allez, je ne vous dis point adieu. Puisse à vos jeunes fronts le souffle moderne plus mortel chaque jour pour les êtres de fantaisie et de rayons que nous fûmes, nous les fées des vieux contes, puisse-t-il vous baigner d'une caresse vivifiante, sous laquelle des songes et des désirs plus beaux fleuriront. (Elle fait un signe d'adieu) ${ }^{45}$.

Dans l'ombre portée d'une pensée si désenchantée, la suite de la pièce s'emploie à développer, dès lors, la partie dysphorique de cette "Belle au bois dormant» inversée. Le deuxième acte le plus long de la pièce est celui de la désillusion et de l'échec de l'aventure «moderne» de la Belle au bois dormant, dans le mouvement d'une prise de conscience malheureuse, où la petite princesse croit découvrir que la réalité qui a pris momentanément les formes de son rêve n'est qu'un leurre. Dans un décor modern style, et sous le regard malveillant d'une «liseuse» de conte — qui n'est autre que la Fée Taciturne - se joue ni plus ni moins qu'un adultère mental, dont le Prince ("la vie jalouse du rêve») va surprendre la scène, comme dans le meilleur vaudeville. Poussée par la tentatrice qui lui rappelle le passé et la rencontre du Prince des songes auquel elle est liée de toute éternité, la Belle, hantée dès lors par le souvenir de son rêve, dans une scène de pure fantasmagorie ${ }^{46}$, consent à la rencontre du fantôme, incube terrifiant, nourri par ses propres pensées, et dont le discours empreint d'un

44. Ibid., acte I, scène 4 .

45. Ibid., acte I, scène 5 . Je souligne.

46. Cette scène mentale, dans sa tentative d'ouverture de la scène intérieure, évoquait le deuxième acte de Madame la Mort, "drame cérébral ", de Rachilde (représenté le 20 mars I89I au théâtre d'Art de Paul Fort), la trilogie d'Antonia d'É. Dujardin, «légende dramatique» en trois 
érotisme morbide et cérébral l'attire irrésistiblement ${ }^{47}$. Au troisième acte, il ne reste au Prince malheureux, qui a surpris cette scène scabreuse, qu'à engager avec l'aide de la bonne marraine le prompt rendormissement de la Belle infidèle : dans le château du début, et à nouveau sur un air de féerie, la Belle ignorante de son sort retrouve sa garde-robe surannée dont elle s'empresse d'extraire une robe rouge avec un pavot sur la poitrine. Une dernière fois, la fée tente de fléchir la volonté du Prince, mais il demeure inébranlable. Activant alors l'ancien charme, la Fée replonge la Princesse dans son sommeil millénaire. Elle tente en vain d'empêcher le Prince d'aller la contempler encore une fois, mais ce dernier, n'y résistant pas, retourne sur ses pas pour donner à sa Belle un dernier baiser, geste dans lequel il se trouve figé à son tour pour l'éternité, ayant voulu, comme un nouvel Orphée, se retourner sur le seuil d'un royaume interdit aux vivants. Ainsi s'achève la féerie de Bataille et d'Humières, sur l'image des deux amants, réunis enfin dans le mystère — «les statues symboliques du rêve», précise la didascalie - tandis que la fée, définitivement dépossédée de ses pouvoirs, exilée du monde, s'en va rejoindre quelque étoile lointaine.

Si un Jean Lorrain peut se réjouir d'une fable si délicatement morbide $^{48}$, les artifices de cette version théâtrale du motif de l'impossible réveil - la lourdeur explicative du texte, la longueur des tirades, le caractère fabriqué de l'intrigue, le luxe et le clinquant de la mise en scène sont autant de défauts que relèvent les critiques des revues symbolistes, dénonçant les maniérismes d'un texte sous influence ${ }^{49}$ et le décorativisme

pièces représentées entre I89I à 1893 (et que Bataille avait vues) et L'Image de M. Beaubourg, drame idéaliste en trois actes qui venait d'être représenté au théâtre de l'EEuvre (27 février I894).

47. H. Bataille et R. d'Humières, ouvr. cité, acte II, scène 3 : «Je veux être pour toi plus qu'une volupté, plus qu'une luxure. J'ai quitté le paradis d'un néant aromal... songe quelle créature je suis! dont chaque atome est de l'amour... Je n'ai ni d'autre but, ni d'autres missions que d'être à toi... Le jour, je disparaîtrai pour me recueillir dans le mystère, et chaque nuit, comme les autres, mais cette fois vivifié, je reviendrai vous abîmer dans les béatitudes. [...] Je suis le fils du rêve, et l'amant et le maître."

48. J. Lorrain, Poussières de Paris, Paris, Fayard, s.d., p. I3-I4 : «Il y avait pourtant de jolies choses dans ces trois actes, une curieuse tentative artiste dans le choix des costumes et des décors; et puis vraiment l'idée de cette Belle au bois qui, durant mille ans, a rêvé d'un autre que de celui qui l'éveille, se désole de ne pas l'avoir rencontré dans la réalité et commet avec sa chimère l'inévitable adultère moral, cette idée-là était charmante avec le dénouement presque shakespearien du mari outragé ramenant la Belle dans son château en ruines pour s'y rendormir à jamais, et, une fois la princesse endormie, ne pouvant l'abandonner tant il l'aime, et renonçant à la vie pour partager avec la morte-vivante son éternel sommeil.»

49. "La Belle au bois dormant, par MM. Bataille et d'Humières, nous révèle deux tempéraments de collectionneurs. Rien que l'idée, une belle idée j'en conviens, rien dans cette pièce n'appartient aux auteurs. Pour l'écriture, elle est empruntée aux livres des symbolistes et tout particulièrement à M. de Régnier. Pour le théâtre, on semble avoir utilisé, les unes après les autres, toutes les audaces 
esthétisant d'un "opéra manquéso ", où se trouvait dévoyée l'idée théâtrale symboliste et trahie la nouveauté des conceptions scéniques inaugurées sur les scènes symbolistes :

Lugné-Poe, en jouant au Théâtre de l'CEuvre La Belle au bois dormant, s'est trompé. [...] Il fallait éblouir, changer, montrer que l'CEuvre, prodigue de régal d'art jusqu'à la féerie d'art, pouvait donner à ses abonnés, nombreux maintenant et de plus en plus exigeants, le luxe d'un princier décor, une fête des yeux, et il en a oublié nos âmes! [...] Écrit sur l'air d'il était une fois... ce pourrait être sublime. Malheureusement, la simplicité n'est pas à la portée de tous. La féerie de MM. Bataille et d'Humières représente la pièce mondaine par excellence; c'est travaillé par un peintre de salon et un officier de cavalerie; c'est du pathos convenable, policé, élégant, très étoffe de chez Liberty; il y a des vers pâles et des phrases pour tous les goûts snobs. J'ai rencontré la phrase triste et sans raison de Maeterlinck, moins sa profondeur d'eau verte; le trait à l'Oscar Wilde, moins l'esprit; la naïveté de Dujardin, moins sa fraîcheur; la joaillerie de Jean Lorrain, mais bien plus fausse; les subtilités de Catulle Mendès, mais moins subtiles; jusqu’à des aphorismes de Victor Hugo, furieusement posthumes par exemple ${ }^{\text {sI }}$

\section{Maeterlinck, ou l'impossible féerie}

Variation plus lointaine sur le motif de la «Belle endormie», dont le sens est radicalement infléchi, la petite féerie tragique des Sept Princesses ${ }^{52}$ de Maurice Maeterlinck donne une expression théâtrale, esthétique et philosophique, d'une toute autre portée, aux préoccupations des symbolistes. Elle occupe de fait une place non négligeable dans les discussions théoriques des contemporains débattant d'un répertoire théâtral pour le symbolisme ${ }^{33}$, alors même que le dramaturge belge devait plus tard la renier,

ou novations qui furent pauvrement essayées au théâtre d'Art. Au Théâtre de l'Euvre l'argent ne manquait point, et l'on en profita pour faire somptueux; mais, cher M. Rochegrosse! c'est de la somptuosité foraine que vous nous confectionnez là! à tort et à travers, comme une simple Loïe Fuller, vous tapez dans la couleur orchestrée; c'est du criard et de la dissonance injustifiée. Il y a pourtant des Principes d'orchestration ou d'harmonie qui, de vous connus, vous conseilleraient peut-être de plus sobres emplois de la lumière colorée. [...].» (P.-N. Roinard, "Théâtre de l'Euvre", Les Essais d'art libre, mars i894, p. II6)

50. Rachilde, "Théâtres: Théâtre de l'Euvre — La Belle au bois dormant", Mercure de France, juillet I894, p. 280.

5I. Ibid.

52. M. Maeterlinck, Les Sept Princesses, Bruxelles, Lacomblez, I89I.

53. Camille Mauclair y voyait la plus symboliste des pièces de Maeterlinck et un quasi-dialogue philosophique : voir "L'art de Maurice Maeterlinck" (Essais d'art libre, février I892, p. 17-26) et "Notes sur un essai de dramaturgie symbolique» (La Revue indépendante, mars 1892, p. 305-17). Voir également Saint-Antoine [H. Mazel], «Le Théâtre symboliste», L’Ermitage, septembre I894, p. I52-I55. 
d'une certaine façon, en l'écartant de l'édition de son Théâtres4 de I90I; et ainsi, la pièce jamais rééditée disparut du répertoire maeterlinckien, où elle semblait pourtant s'inscrire dans l'ensemble de son premier théâtre, à côté des autres pièces légendaires, auxquelles elle est liée thématiquement et comme "inter-fictionnellement ${ }^{55}$ ». La pièce a donné lieu à un spectacle de marionnettes, réalisé dans la mouvance des peintres nabis, lors d'une séance privée organisée par Paul Ranson, chez le conseiller d'État Georges Coulon, le dimanche ro avril $1892^{56}$ : costumes de Maurice Denis, décors de Vuillard, programme de Ranson, rideau de scène de Jan Verkade, lecture de Sérusier - pour une «représentation d'art» mémorable, en dépit du caractère éphémère de cette séance privée, et qui correspondait au souhait du dramaturge de voir son théâtre joué par des marionnettes, afin d'en préserver la poésie singulière et l'effet de suggestion ${ }^{57}$.

Empruntant volontiers à l'univers des contes - moins ceux de Perrault et de la tradition du "Cabinet des fées" qu'il jugeait trop rationnelle et savante, qu'aux contes de Grimm auxquels il vouait une prédilection particulière ${ }^{58}$ - Maeterlinck y joue de références multiples, avec un art consommé de la réminiscence. Mais tandis que les héros de «BlancheNeige», du "Cercueil de verre», ou de la «Belle au bois dormant», sont des libérateurs qui parviennent à délivrer les belles de leur sommeil enchanté, le prince Marcellus intervient ici à contretemps, et sa venue coïncide avec la mort de l'élue : la traversée du souterrain — épreuve à laquelle il est soumis, conformément à la loi des contes — se révèle inefficace,

54. M. Maeterlinck, Théâtre, avec une préface inédite de l'auteur, Bruxelles, Deman, Lacomblez, I9oI-1902, 3 vol. [T. I : La Princesse Maleine, L'Intruse, Les Avengles; T. II : Alladine et Palomidès, Intérieur, La Mort de Tintagiles; T. III : Aglavaine et Sélysette, Ariane et Barbe-Bleue, Béatrice].

55. Notamment à travers certains effets citationnels de l'onomastique : dans Pelléas et Mélisande, composé un an après l'acte des Sept Princesses, l'on apprend à l'acte I, qu'Arkël aurait voulu marier Golaud à une Princesse Ursule; et aux actes II et III, que Pelléas doit se rendre au chevet de son ami Marcellus.

56. Voir Artistes et Théâtres d'Avant-Garde. Programmes de Théâtre illustrés Paris 1890-1900, G. Aitken (éd.), catalogue de L'Expul, Musée de Pully, Musée de Marmottant, 1991, p. 95; voir aussi J.-P. Bouillon, Maurice Denis (I870-1943), ouvr. cité, p. 23.

57. Voir M. Maeterlinck, «Menus propos : le théâtre (Un théâtre d'Androïdes) ", La Jeune Belgique, septembre I890, p. 331-336 (repris dans M. Maeterlinck, Introduction à une psychologie des songes et autres écrits I886-I896, Bruxelles, Éditions Labor, 1985), et Trois petits drames pour marionnettes [Alladine et Palomidès, Intérieur, La Mort de Tintagiles], avec des culs-de-lampe dessinés et gravés sur bois par G. Minne, Bruxelles, Deman, I894; repris dans Théâtre, t. II, Bruxelles, Deman, 1902. 58. Voir le Cahier bleu (Éditions de la Fondation Maeterlinck, 1977), p. I35 : «Art germanique. Comparez le fantastique des contes de Grimm et de la mort d'Arthur, eux animent tout [...] et les contes de Perrault qui tâchent d'être explicables et vraisemblables, ayant peur de tout - très restreint.» 
et le Prince charmant n'arrive que pour constater la mort de la Belle. $\mathrm{Au}$ rebours de la dynamique des contes de fées, Maeterlinck livre ainsi à nouveau l'une de ces féeries tristes, dans lesquelles, depuis La Princesse Maleine, il traite le conte théâtral en tragédie métaphysique. Les ingrédients thématiques et textuels du malaise maeterlinckien y sont à l'œuvre : fragilité constitutive d'une humanité d'enfants et de vieillards perdue dans un monde en déréliction que les fées ont déserté, dialogues balbutiants, répétitions, phrases inachevées, allusions énigmatiques, suspens, silences, prémonitions ${ }^{59}$... Et comme dans L'Intruse ou dans Les Aveugles, la pièce se développe en un acte unique, où l'essentiel de l'action consiste à montrer, à travers l'angoisse des personnages (la vieille Reine) et des signes "atmosphériques" funestes, le travail rampant de la mort qui vient frapper l'un des représentants de cette humanité impuissante. Le sommeil de l'âme et ses réveils problématiques, ses alternances de longues léthargies et de reviviscences, toutes ces images sont au centre de la pensée spiritualiste de Maeterlinck ${ }^{60}$, et fournissent une clef d'interprétation de son théâtre, celui $\mathrm{du}$ "tragique quotidien» et celui de la réconciliation. Parmi les drames légendaires de son premier théâtre, Les Sept Princesses illustre tout particulièrement les conceptions mystiques de Maeterlinck, en faisant du réveil problématique des jeunes filles endormies le motif central de la fable, ainsi que son principal enjeu. Dans son travail de réinvention légendaire, et en recourant aux procédés traditionnellement symboliques du merveilleux, le dramaturge met en avant le caractère énigmatique d'une fable théâtrale destinée à évoquer les opérations mystérieuses de la vie spirituelle. Ainsi le personnage de la belle endormie se trouve-t-il démultiplié, selon le principe d'une symbolique des nombres (le chiffre sept correspond également au nombre d'années que le prince a passées loin du château), susceptible de renvoyer aussi bien aux contes populaires qu’à la tradition chrétienne (faisant signe par exemple du côté de la Légende dorée de Voragine et de l'histoire des "Sept dormants $\left.{ }^{61} »\right)$. Si au cours de la pièce, les individualités des sept petites cousines malades du prince Marcellus sont fugace-

59. Voir Ch. Lutaud, "Les Sept Princesses ou la mort maeterlinckienne», dans Les Lettres romanes, "Le Centenaire du Symbolisme en Belgique», Louvain, n 3-4, 1986, p. 255-273.

6o. Voir dans Le Tragique quotidien, le chapitre consacré au «Réveil de l'âme», un article originellement publié dans La Nouvelle Revue, mai-juin I895, t. 94, p. 493-509, sous le titre de «Lâme se réveille».

6I. Voir l'histoire des "Sept dormants», une légende chrétienne rapportée par Jacques de Voragine dans sa Légende dorée qui évoquait le sommeil de trois cent soixante-dix-sept ans des sept saints d'Éphèse, persécutés sous l'empereur Dèce et miraculeusement réveillés sous le règne de Théodose. 
ment évoquées par la mention de leurs prénoms ${ }^{62}$, et si la princesse Ursule gagne finalement le statut unique de victime tragique, il n'en demeure pas moins que la pièce impose, ne serait-ce que par son titre, l'unité du mystérieux symbole pluriel que constitue le groupe des «sept princesses», et que l'interprétation symbolique de ce collectif importe plus par l'image qu'il impose tout au long de la pièce, que les particularités de la fable imaginée par le poète.

L'intérêt des Sept Princesses — sa bizarrerie dramaturgique aussi réside essentiellement, en effet, dans la manière dont Maeterlinck a choisi de traiter spatialement son drame en définissant un dispositif scénique complexe qui commande la conduite de l'action et lui confère sa signification symbolique : au premier plan, la salle de marbre et ses sept marches étagées où dorment les princesses; une porte close et deux hautes fenêtres délimitent, à l'arrière de la scène, un second plan — celui de la terrasse où se tiennent les vieux souverains et le prince Marcellus, lesquels, à défaut de pouvoir pénétrer dans la salle, observent ce qui se passe par les grandes vitres. Le fonctionnement d'un tel dispositif, longuement décrit dans la didascalie initiale ${ }^{63}$ - et qui constitue d'abord le tableau des belles endormies en une vision énigmatique - parait problématique, voire assez impraticable, à la mise en scène : soit, en effet, il faut imaginer une présentation préalable de ce beau décor floral et virginal, offrant au spectateur une première contemplation silencieuse des princesses endormies, avant que, par le moyen de quelque impressionnant changement à vue, le décor ne tourne à cent quatre-vingt degrés pour obéir au changement de point de vue induit par la situation de la première scène qui se déroule sur la terrasse; soit, et cela paraît plus probable, Maeterlinck, sans souci de logique ni de vraisemblance scéniques, a privilégié le symbole, en imaginant un dispositif étagé, où la position surélevée des personnages sur la terrasse suffirait à faire fonctionner l'ensemble (le spectateur, placé du côté des

62. «LA ReIne : Les reconnaissez-vous, — Voilà Geneviève, Hélène et Christabelle... et de l'autre côté, voilà Madeleine, Claire et Claribelle avec des émeraudes [...].» (M. Maeterlinck, Les Sept Princesses, ouvr. cité, p. 25)

63. Ibid., p. 6 : «Une vaste salle de marbre, avec des lauriers, des lavandes et des lys en des vases de porcelaine. Un escalier aux sept marches de marbre blanc divise longitudinalement toute la salle, et sept princesses, en robes blanches et les bras nus, sont endormies sur ces marches garnies de coussins de soie pâle. Une lampe d'argent éclaire leur sommeil. Au fond de la salle, une porte aux puissants verrous. À droite et à gauche de la porte, de grandes fenêtres dont les vitrages descendent jusqu'au ras du carrelage. Derrière ces fenêtres, une terrasse. Le soleil est sur le point de se coucher et l'on aperçoit, à travers les vitrages, une noire campagne marécageuse avec des étangs et des forêts de chênes et de pins. Perpendiculairement à l'une des fenêtres, entre d'énormes saules, un sombre canal inflexible, à l'horizon duquel s'avance un grand navire de guerre.» 
princesses, observerait et entendrait donc les personnages principaux de l'autre côté des vitres, au fond de la scène, dans une position symétrique et inverse à la leur?). Faut-il conclure de la réelle difficulté posée par un tel dispositif, que Maeterlinck destinait exclusivement son drame à la lecture? Rien n'est moins sûr. Car il y revient avec succès dans Intérieur, en I894, en imaginant cette fois une véritable tragédie du regard, à laquelle le spectateur est en mesure de collaborer plus activement ${ }^{64}$. Ainsi séparées par un mur de verre des trois personnages principaux - et des spectateurs de la salle, de manière techniquement improbable - les sept princesses sont placées comme dans une vitrine, ou une chasse, ou encore comme dans une "serre» - pour reprendre une image chère à Maeterlinck —, offrant une image muette, inquiétante autant que belle, à ceux qui les observent. Après une mystérieuse traversée souterraine qui lui permettra de franchir l'obstacle (de franchir le mur des apparences?), seul le prince, à la fin de la pièce, sera autorisé à pénétrer ce lieu de clôture, qui apparaît presque comme un endroit sacré. Cette traversée d'un espace de mort (le caveau des aïeux) constitue ainsi l'épreuve nécessaire pour accéder au lieu interdit, le terme d'un parcours initiatique. Mais si initiation il y a, elle se révèle déceptive, puisqu'au bout du chemin Marcellus est confronté à la mort de l'une des princesses, sa préférée, qui ne se réveillera plus. La partition de l'espace paraît ainsi figurer scéniquement ce «théâtre double» qui hantait Maeterlinck, offrant l'image concrète de la superposition de deux réalités, selon une conception mystique distinguant à côté (au-dessus?) de la réalité (celle du corps et de la vie sensitive), une réalité supérieure, lieu de vérité, de connaissance, d'illumination (la sphère d'appartenance de l'âme) — le prince, à plusieurs reprises, parle de la lumière dans la salle de marbre —, où l'on parviendrait par une ascèse, par une initiation : dans une telle perspective, le drame de Maeterlinck semble relever d'une conception néo-platonicienne, opposant la réalité sensible et le monde des idées; s'il est loisible à l'homme de s'élever de l'une à l'autre, par la contemplation, sa condition mortelle pourtant limite l'accès du monde intelligible qui toujours se dérobe à lui, ainsi que le suggère la quête vaine du prince. Pour Camille Mauclair, qui s'intéressait à l'idéalisme mystique de Maeterlinck, il s'agissait là ni plus ni moins que de l'actualisation scénique de la philosophie de Schopenhauer qui comparait le monde sacré des idées à une forteresse autour de laquelle un guerrier tournerait en vain, et au cœur de laquelle on ne pourrait accéder que par un souterrain.

64. M. Maeterlinck, Intérieur, Trois petits drames pour marionnettes, ouvr. cité; repris dans Théâtre, t. II, ouvr. cité. Représenté au Nouveau Théâtre par le théâtre de l'CEuvre, le I5 mars I895. 
Marcellus était bien ce guerrier qui renonçait d'abord à l'action, comme le suggérait au début de la pièce l'évocation du navire de guerre qui s'éloignait et le chant des matelots disant qu'ils ne reviendraient plus. Le prétexte fictionnel du retour du jeune prince était la quête de sa fiancée, mais symboliquement il fallait y voir l'image de l'âme humaine en quête de son rêve, - un rêve et un au-delà qui allaient demeurer inaccessibles au vivant :

[...] le vieux roi lui indique un souterrain qui aboutit au milieu même de la salle où dorment les princesses : il faut passer entre les tombeaux des ancêtres. (Toujours l'idée de la Mort, transition inévitable). Marcellus part; quand il émerge dans la salle, - au cœur de la forteresse - pour posséder enfin son rêve, il faudrait qu'il ne fût plus qu'un esprit, qu'il mourût. Mais il est resté vivant, c'est-à-dire sacrilège; et voici que l'idée profanée s'évanouit. La Princesse est morte, ses sœurs l'emportent tandis que derrière l'impénétrable mirage de la porte de cristal, le roi, la reine, les serviteurs, les humbles comme les puissants, s'épuisent en clameurs inutiles devant l'Invisible. Telle est cette ouvre philosophique, la plus intense, la plus complète, la plus absolue qu'ait conçue M. Maeterlinck. Dégagé résolument des apparences, purifié par l'idée de la mort dont il a su rendre l'épouvante terrestre tout en proclamant sa sublime utilité spirituelle, il est dès à présent sorti du monde réel. Et, le pied posé sur le Territoire, calme héros, il n'a plus qu’à poursuivre l'hymen définitif des princesses ${ }^{65}$.

En dépit de cette admiration de Mauclair — que ne partagent pas tous les symbolistes ${ }^{66}$ - la féerie tragique des Sept Princesses constituait, on l'a vu, un échec pour Maeterlinck lui-même, sans doute à cause de l'insoluble problème scénographique qu'elle posait, mais aussi probablement en raison de son caractère trop ouvertement allégorique. Dans la série théâtrale des "Belles endormies" fin-de-siècle envisagées ici — et pour ces raisons mêmes - elle paraît exemplaire, constituant une sorte de point-limite et un ratage significatif des contradictions d'un théâtre peinant — autour du mythe de la Belle au bois dormant — à articuler la dimension du spectaculaire et celle du mystère.

65. Ibid., p. 24 .

66. Pour Adolphe Retté, par exemple, le chef-d'œuvre de Maeterlinck restait Les Aveugles, tandis que Les Sept Princesses relevait pour lui d'une forme de maniérisme ("Les Poésies», L'Ermitage, janvier I892). De même Henri Mazel (sous son pseudonyme de Saint-Antoine) déplorait «le sens allégorique trop visible» des Sept Princesses, qui en «refroidissait l'intérêt théâtral» ("Le Théâtre symboliste", L'Ermitage, septembre 1894, p. 152-I55). 